\title{
Effets De La Dynamique Du Couvert Végétal Sur Les Écoulements Dans Le Bassin Versant De La Lagune Aghien En Côte d'Ivoire
}

\author{
Diallo Seydou, Doctorant en Géosciences et Environnement \\ Noufé Dabissi, Maître Assistant \\ Université Nangui Abrogoua, Côte d'Ivoire \\ Tra Bi Zamblé Armand, Maître Assistant \\ Université Alassane Ouattara, Côte d'Ivoire \\ Dao Amidou, Maître Assistant \\ Université Nangui Abrogoua, Côte d'Ivoire \\ Kamagaté Bamory, Maître de Conférence \\ Effebi Kôkôh Rose, Maître de Conférence \\ Goné Droh Lanciné, Professeur Titulaire \\ Koffi Ehouman Serge, Doctorant en Géosciences et \\ Environnement \\ Koffi Jean Thierry, Doctorant en Géosciences et \\ Environnement \\ Université Nangui Abrogoua, Côte d'Ivoire \\ Jean Emmanuel Paturel, Chercheur à IRD \\ Jean-Louis Perrin, Chercheur à IRD \\ Luc Seguis, Chercheur à IRD \\ Université de Montpellier, France
}

Doi: 10.19044/esj.2018.v14n36p312 URL:http://dx.doi.org/10.19044/esj.2018.v14n36p312

Abstract

The aim of this paper is to highlight the impact of anthropization on the runoff Djibi and Bete rivers, tributaries of the Ivorian Aghien watershed. The classification method with the maximum likelihood used to the diachronic study of satellite imagery Landsat Thematic Mapper (1987), Enhance Thematic Mapper Plus (2000) and Operational Land Imager (2015) and Geographic Information System remote sensing has been applied. It has permitted to note the total disappearance of the forest at risk of subsistence crops, fallows, habitats and bare soils. The forest area that was 4 ha in 1987 for the Bete Watershed has been destroyed entirely. This change in the land cover between 1987 and 2015 had considerable consequencies on the 
contributions of water to the level of the Djibi and Bete rivers. So bad slight values of the retention capacity indices underneath 100 reflect the anthropization of these watersheds. The use of rainfall series for the evaluation on the runoff from the Vuillaume Dubreuil method (1975), reveals an increase in runoff coefficients from $20 \%$ to $21 \%$ in Djibi watershed and from $13 \%$ to $17 \%$ in Bete watershed between 1987 and 2015. Increasing these flow coefficients related to water in a watershed more and more urbanized inflow is a threat to the water quality and the security of area urbanized.

Keywords: Land use, anthropization, runoff, basins Djibi and Bete

\section{Resume}

Cette étude évalue l'impact de l'anthropisation sur les écoulements des sous bassins Djibi et Bété, tributaires du bassin de la lagune Aghien en Côte d'Ivoire. L'étude diachronique d'images satellitaires Landsat Thematic Mapper (1987), Enhance Thematic Mapper Plus (2000) et Operational Land Imager (2015) à travers la méthode de classification par maximum de vraisemblance et les systèmes d'information cartographique a été appliquée. Elle a permis de constater la disparition totale de la forêt au profit des cultures vivrières, des jachères, des habitats et des sols nus. La surface forestière qui était de 4 ha en 1987 pour le bassin versant de la Bété a entièrement été détruite. Ce changement de l'occupation du sol entre 1987 et 2015 a eu des conséquences notables sur les apports d'eau au niveau des rivières Djibi et Bété. Les indices de capacité de rétention inférieurs à 100 témoignent de l'anthropisation de ces bassins versants. L'utilisation des séries pluviométriques pour l'évaluation des lames d'eau écoulées à partir de la méthode de Vuilllaume-Dubreuil (1975), révèle une hausse des coefficients d'écoulements de $20 \%$ à $21 \%$ sur le bassin versant Djibi et de $13 \%$ à $17 \%$ sur celui de la Bété de 1987 à 2015. L'augmentation de ces coefficients d'écoulements dans un bassin de plus en plus anthropisé constitue une menace pour la qualité des eaux et la sécurité des espaces bâtis.

Mots-clés: Anthropisation, télédétection, écoulements, bassins Djibi et Bété

\section{Introduction}

L'urbanisation dans les grandes villes africaines s'accompagne de préoccupations environnementales majeures à bien des égards. On assiste à une imperméabilisation de la surface des sols dans un contexte de vétusté et de surcharge des ouvrages d'assainissement qui aboutit à de fréquentes inondations. 
L'impact des états de surface et de l'occupation du sol sur les écoulements superficiels a fait l'objet de plusieurs études à travers le monde (Vissin, 2007; Mahé et al., 2010). La plupart des auteurs concluent à l'augmentation des écoulements de surface du fait de l'imperméabilisation des sols (Soro et al., 2006 ; Mahé et al., 2010 ; Soro et al., 2013 ; Yao et al., 2015). Les études hydrologiques en Afrique de l'Ouest ont montré que les coefficients d'écoulement ont fortement augmenté sur certains hydrosystèmes du Sahel, en dépit d'une diminution marquée de la pluviométrie régionale (Cecchi et al., 2009; Mahé et al., 2010). Ce phénomène surnommé «paradoxe du désert» (Capperlaere et al., 2009; Dao, 2013) s’observe davantage dans les zones sahéliennes caractérisées par une diminution du couvert végétal, une extension des surfaces cultivées et des surfaces dégradées. La zone tropicale humide avec une végétation abondante présente des caractéristiques pluviométriques aux antipodes de celles des régions sahéliennes. Toutefois, cette zone semble subir les mêmes effets de la baisse des capacités d'infiltrations en eau des sols, surtout dans les bassins urbains et péri-urbains (Soro et al., 2014).

Situées en zone péri-urbaine sur le littoral Est ivoirien, les rivières Djibi et Bété sont les principaux affluents de la lagune Aghien. Les sous bassins versants de cette lagune sont caractérisés par une urbanisation en extension avec pour corollaire la dégradation des derniers « ilôts » de forêts au profit de l'habitat et des cultures. Ces évolutions ont des répercussions directes sur le fonctionnement hydrologique des bassins versants. En effet, les études consacrées à l'hydrologie de surface en relation avec l'évolution des couverts végétaux des petits bassins versants du littoral ivoirien, à fortiori les sous bassins versants de la Djibi et la Bété dans le District d'Abidjan sont rares. En outre, ces sous bassins avec des surfaces respectives de $78 \mathrm{~km}^{2}$ et de $206 \mathrm{~km}^{2}$ pour la Djibi et la Bété, n'ont même pas fait 1'objet de jaugeages jusqu'à la fin des années 2014 (Projet Aghien, 2014). Ainsi, cette étude a été initiée dans le but de montrer l'évolution du couvert végétal en 1987, 2000 et 2015 à partir des données de télédétection (images Landsat TM, ETM+ et OLI) dans les dits bassins versants et leur impact sur les coefficients d'écoulement. La mise en évidence des phénomènes de ruissellement et d'infiltrations dans ce travail a eu pour support les types d'occupation du sol de ces bassins versants et l'évaluation des écoulements moyens annuels à partir de la méthode de Vuillaume-Dubreuil (1975). Les classes d'occupation du sol obtenues par classification des images Landsat ont été affectées de coefficients de pondération représentant leur capacité de rétention en eau (Cecchi et al, 2009 ; Soro et al, 2014). L'évolution des coefficients de rétention en 1987, 2000 et 2015 des sous bassins versants Djibi et Bété, respectivement associée aux informations des postes pluviométriques du Centre National de Recherche Agronomique de Bingerville et de IRHO Lamé, 
ont permis d'analyser l'impact de la modification du couvert végétal sur les écoulements de surface.

\section{Zone d'étude, données et méthodes}

\subsection{Localisation de la zone d'étude}

Les rivières Djibi et Bété sont les principaux tributaires du bassin ivoirien de la lagune Aghien. Elles sont situées au Nord-Est de la ville d'Abidjan (capital économique de Côte d'Ivoire). Elles sont limitées au Nord par la sous-préfecture d'Azaguié et au Sud par celle de Bingerville (Figure 1).

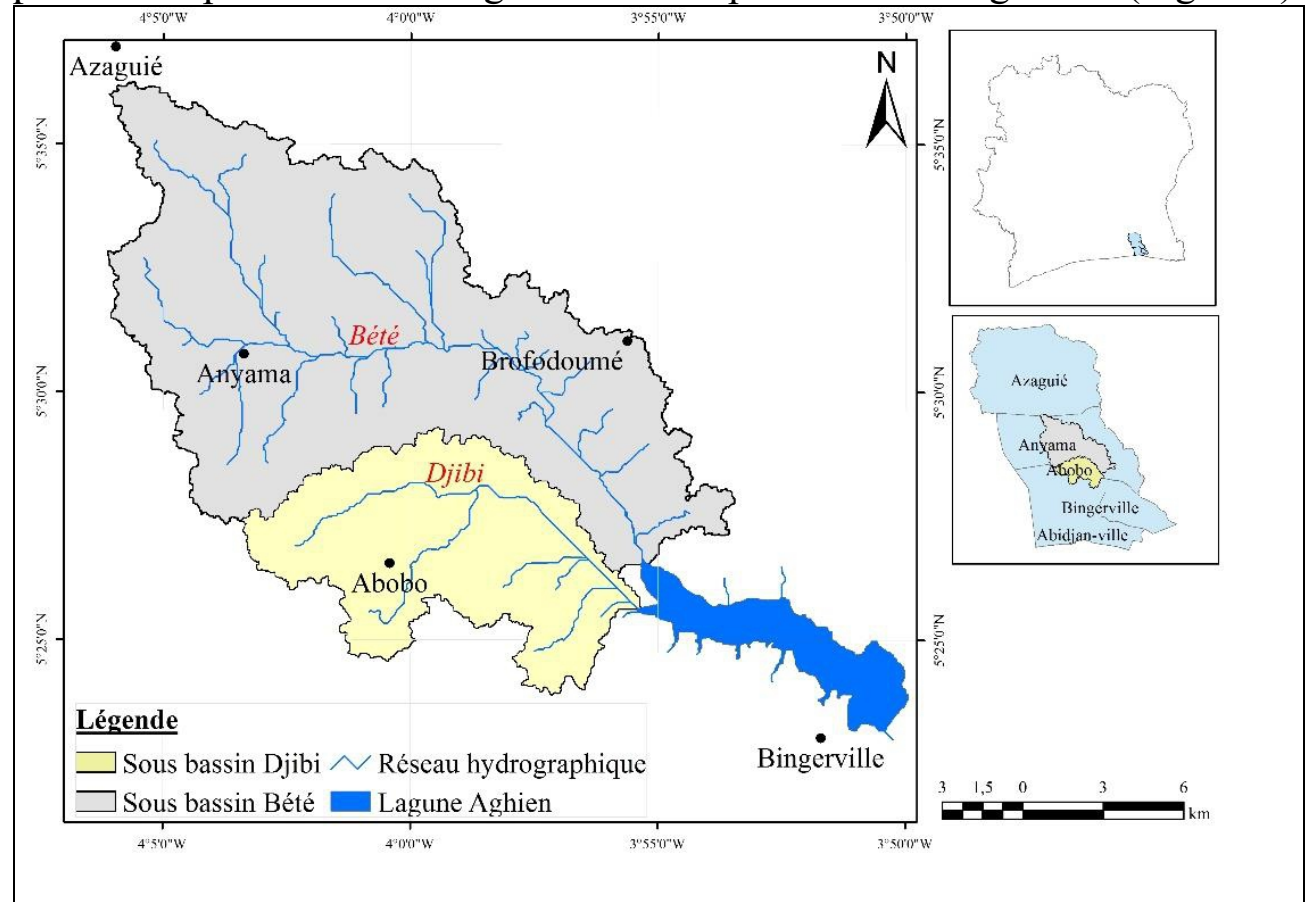

Figure 1 : Localisation de la zone étudiée (source : adapté du projet Aghien, 2014)

Les bassins versants Djibi et Bété traversent les périphéries de deux localités urbaines (Abobo et Anyama) et ont respectivement pour superficies $78 \mathrm{~km}^{2}$ et $206 \mathrm{~km}^{2}$ et pour périmètres $77 \mathrm{~km}$ et $135 \mathrm{~km}$ (Tableau 1). Ces bassins ont une forme allongée car les indices de compacité de Gravelius sont supérieurs à 1,2 (2,46 pour le bassin de la Djibi et 2,65 pour la Bété). Dans ces bassins, les altitudes varient de 12 à $137 \mathrm{~m}$.

Tableau 1 : Caractéristiques géométriques des bassins versants Djibi et Bété

\begin{tabular}{lll}
\hline Caractéristiques Physiques & bassin Djibi & bassin Bété \\
\hline Surface $\left(\mathbf{k m}^{\mathbf{2}}\right)$ & 78 & 206 \\
\hline Périmètre $(\mathbf{k m})$ & 77 & 135 \\
\hline Altitude minimale $(\mathbf{m})$ & 12 & 12 \\
\hline Altitude maximale $(\mathbf{m})$ & 135 & 137 \\
\hline
\end{tabular}




\begin{tabular}{lll}
\hline Altitude moyenne (m) & 49,25 & 64,77 \\
\hline Indice de compacité de Gravelius (m) & 2,46 & 2,65 \\
\hline Dénivelée (m) & 100 & 66 \\
\hline Indice global de pente (m/km) & 2,75 & 1,03 \\
\hline Dénivelée spécifique (m) & 24,29 & 14,78 \\
\hline Longueur du rectangle équivalent $(\mathbf{k m})$ & 36,35 & 64,3 \\
\hline Largeur du rectangle équivalent $(\mathbf{k m})$ & 2,15 & 3,2
\end{tabular}

Au regard du tableau ci-dessus Les dénivelés spécifiques des bassins versants sont en dessous de $25 \mathrm{~m}$, ce qui nous amène à dire que l'altitude du relief est faible selon la méthode de classification du relief de l'IRD (Institut de Recherche pour le développpement) ex ORSTOM.

Le socle géologique est un ensemble formé de deux unités dont le bassin sédimentaire au sud et le socle cristallin et cristallophyllien dans la partie nord de la Djibi et de la Bété (Jourda, 1987).

Le climat de la région est de type équatorial de transition avec deux saisons pluvieuses (une grande et une petite saison de pluies respectivement d'Avril à Juin et de Septembre à Novembre) et deux saisons sèches (une grande saison sèche de Décembre à Mars et une petite saison sèche de Juillet à Août) (Soro, 2004). La pluviométrie moyenne annuelle est généralement supérieure à $1500 \mathrm{~mm}$. L'agriculture est la principale activité économique sur ces bassins. Les cultures d'exportations telles que le palmier à huile, l'hévéa, le café, le cacao sont plus abondantes dans le bassin de la Bété tandis que les cultures vivrières (le maïs, le manioc, la banane plantain) sont plus cultivées dans celui de la Djibi (Bondy, 2015).

\subsection{Données de l'étude}

Dans le cadre de cette étude, nous avons utilisé des données satellitaires et pluviométriques. Les données satellitaires disponibles gratuitement sur le site internet earth explorer sont issues de la National Aeronautic and Space Administration. Les séries pluviométriques quant à elles proviennent de la Direction de la Météorologie Nationale de la Côte d'Ivoire. Pour l'analyse de la dynamique du couvert végétal, nous avons eu recours à l'imagerie satellitaire des capteurs Landsat. Ces images ont une résolution spatiale de $30 \mathrm{~m}$ et concernent les dates du 22 décembre 1987, du 02 au 09 février 2000 et du 27 décembre 2015. Ces dates ont été choisies en référence aux trois derniers recensements nationaux de la population et de l'habitat, car l'évolution de cette population est un moteur important du changement de l'occupation du sol. Ces images prises toutes en saison sèche (décembre et février) permettent une bonne discrimination des unités d'occupation du sol, parce que c'est la période pendant laquelle les taux de couverture nuageuse 
sont les plus faibles (Chatelain, 1996 ; Barima et al., 2009 ; Kouassi, 2014). Par ailleurs, ce choix contribue à diminuer d'éventuels effets saisonniers.

En plus de ces images Landsat, nous avons utilisé un Modèle Numérique de Terrain (MNT) issu de la base des données Shuttle Radar Topography Mission (SRTM) de 30 mètres de résolution spatiale également.

Concernant les séries pluviométriques annuelles, elles s'étendent de 1960 à 2017. Cette période couvre les différentes dates retenues pour l'analyse de la dynamique spatio-temporelle du couvert végétal. Les données pluviométriques et celles d'EvapoTranspiration Potentielle (ETP) utilisées au pas de temps mensuel sont celles des postes pluviométriques CNRA Bingerville et IRHO LaMé.

\subsection{Méthodes}

\subsubsection{La cartographie de l'occupation du sol des bassins Djibi et Bété}

La méthode de comparaison des différences de classe entre les dates de 1987-2000, 2000-2015 et 1987-2015 a été utilisé pour une analyse du changement du couvert végétal. Les images Landsat utilisées étaient déjà orthorectifiées et projetées dans le système UTM, WGS84, zone $30 \mathrm{~N}$. Une correction géométrique n'a donc pas été nécessaire. Toutefois, pour permettre une bonne comparaison des classes d'occupation du sol aux différentes dates, les effets atmosphériques ont été réduits à travers des corrections atmosphériques pour chaque image. Pour une bonne discrimination des types de végétation et sa distinction avec l'espace bâti, la combinaison colorée infrarouge moyen 1 (bande 5 de Landsat TM et ETM+, bande 6 de Landsat OLI)-proche infrarouge (bande 4 de TM et ETM+ et bande 5 de OLI)-rouge (bande 3 de TM et ETM+ et bande 4 de OLI) a été utilisée. Sept classes d'occupation de sol ont ainsi été retenues :

eau, cultures vivrières et jachères, localités (habitat concentré), localités (habitat dispersé) et sol nu, palmeraie + plantations + forêt dégradée, Hévéa + plantations + forêt dégradée et Forêt en zone humide.

La méthode de classification adoptée est celle du maximum de vraisemblance (Tra Bi, 2013 ; Coulibaly et al., 2016). Cette méthode calcule selon la réflectance des pixels, la probabilité de leur appartenance à une classe donnée. Le pixel sera affecté à la classe pour laquelle la probabilité est la plus forte. Cependant, si cette probabilité n'atteint pas le seuil escompté, le pixel est classé "inconnu".

Enfin, un filtre majoritaire avec une fenêtre $3 \times 3$ a été appliqué pour homogénéiser les rendus cartographiques. Cette opération a pour but d'affecter le pixel central du filtre à la classe la plus représentée. Une évaluation des pourcentages des différents types du couvert végétal et des états de surface a servi à analyser l'évolution des différents éléments de l'occupation du sol des bassins versants Djibi et Bété en 1987, 2000 et 2015. 
Les relevés sur le terrain ont permis de valider cette classification. A partir de la visite de terrain de ces deux bassins, une description de ces zones a été faite sur la base des parcelles sélectionnées.

Le taux d'évolution des classes de l'occupation du sol a été utilisé pour exprimer la variation de l'occupation du sol entre deux dates. Selon Coulibaly, 2016, ce taux a été calculé par la relation 1 suivante $T=\frac{V_{2}-V_{1}}{V_{1}}$ (1)

Avec $T$ : Taux d'évolution du couvert végétal entre les deux dates d'observation ;

$V_{1}$ : La proportion de la classe de l'occupation du sol prise à l'état initial ; $V_{2}$ : La proportion de la classe de l'occupation du sol prise à l'état final.

\subsubsection{Détermination d'indice de capacité de rétention en eau des types d'occupation du sol}

Pour évaluer l'impact de la dynamique du couvert végétal sur les écoulements, un indice représentant la capacité de rétention en eau pour chaque classe d'occupation du sol est calculé pour l'année considérée (Cecchi et al., 2009 ; Soro et al., 2014). Il s'exprime par l'équation (2) suivante : $\mathrm{Cr}=$ $\sum_{i} P i \times a i$

Avec $P i$ : pourcentage en superficie de la zone d'étude occupée par la classe d'occupation du sol $i$;

ai: coefficient de pondération indiquant la capacité de rétention en eau effective de la classe $i$,

Pour chaque classe, le coefficient ai (Tableau 2) est représenté par une valeur comprise entre 0 et 2 , une valeur de zéro représentant «une rétention nulle» et une valeur de deux représentant « une rétention maximale » (Cecchi et al., 2009 ; Soro, 2010). Quant au coefficient Cr, il est compris entre 0 et 200 et s'applique sur des bassins de petites tailles pour discriminer avec efficacité les différentes classes d'occupation du sol par télédétection $(>25$ $\mathrm{km}^{2}$ ). Les valeurs élevées (>100) correspondent alors à des bassins « sauvages » (non maîtrisés) dotés d'une importante couverture végétale, d'une forte capacité de rétention en eau et donc peu propice à l'intensification des écoulements, tandis que les valeurs faibles correspondraient à des bassins anthropisés, voire dégradés, dotés d'une faible capacité de rétention et donc à l'inverse des précédents, propices à d'importants écoulements. Pour cette étude, la codification établie a été faite sur la base des études de Cecchi et al., (2009) et des cotes attribuées aux classes d'occupation du sol (Soro, 2010 ; Soro et al.,2014). L'accent a été mis sur la capacité de rétention en eau du bassin par la végétation ou sur l'efficacité du bassin à accélérer l'écoulement favorisé par l'anthropisation. Plus le couvert végétal est dense et moins la zone est anthropisée c'est-à-dire propice à un faible écoulement et inversement. Ainsi, la valeur 2 a été attribuée à la forêt à cause de la plus grande densité du couvert végétal. Son existence sur le bassin empêche ou réduit l'intensité de 
l'écoulement. Les coefficients 0 et 1 qui représentent les plus faibles valeurs ont été attribués respectivement aux habitats concentrés et habitats dispersés (tableau 2). En effet, la pondération 1 a été affectée à la classe habitat dispersé, car l'espace qu'elle occupe est un mélange habitats et de végétation.

Tableau 2: Coefficient de capacité de rétention des classes d'occupation du sol étudié

\begin{tabular}{ll}
\hline Classes d'occupation du sol & ai \\
\hline Habitats concentrés & 0 \\
Habitats dispersés & 1 \\
Cultures vivrières et jachères & 1 \\
Cultures de rente : Hévéa et Palmier & 1 \\
Forêt & 2 \\
\hline
\end{tabular}

\subsubsection{Estimation des lames d'eau écoulées par la méthode de Vuillaume- Dubreuil}

En Afrique occidentale, la méthode utilisée pour l'évaluation des écoulements sur des bassins non jaugés est celle de Vuillaume-Dubreuil (Dubreuil et Vuillaume, 1975). La méthode prend en compte la notion de pluie efficace qui correspond à la pluie qui contribue à l'écoulement. L'écoulement moyen annuel des bassins versants non jaugés est déterminé à partir de leurs caractéristiques physiques et climatiques. Elle est applicable entre les isohyètes 400 et $4200 \mathrm{~mm}$ de hauteur pluviométrique moyenne annuelle (Dubreuil et Vuillaume, 1975) pour des bassins dont la superficie se situe entre quelques dizaines et plus de $100 \mathrm{~km}^{2}$. De l'avis des auteurs, elle peut être étendue à l'intervalle 1 à $1000 \mathrm{~km}^{2}$. Le facteur explicatif principal $P_{r}$ est un facteur climatique qui représente la part disponible pour l'écoulement de l'apport pluvial considéré à l'échelle mensuelle. La partie non disponible étant sensée être représentée par l'évapotranspiration, c'est-à-dire la partie de l'eau précipitée qui ne rejoint ni les cours d'eau, ni les nappes souterraines, mais qui s'évapore.

La part de la pluie qui n'est pas évaporée, constitue la pluie efficace qui contribue à l'écoulement. Pour Dubreuil et Vuillaume en 1975, cette part peut être calculée par le biais d'une pluie réduite définie par l'équation (3) suivante : $\operatorname{Pr}=\sum_{n-1}^{12} \delta^{n}\left(P_{n}-\frac{E T B}{36}\right)$

Avec :

$\delta^{n}=1$ quand $P_{n} \geq \frac{E T B}{36}$

$\delta^{n}=0$ quand $P_{n} \leq \frac{E T B}{36}$

$n$ : varie de 1 à 12 (mois)

$P_{r}$ : apport pluvial après l'évapotranspiration $(\mathrm{mm})$ 
$P_{n}$ : pluie totale mensuelle du mois $\mathrm{n}$

$E T B$ : Evaporation annuelle moyenne sur bac d'eau libre (mm) avec $E T P=0,75$ ETB,

Par la même occasion, la relation (4) proposée par Dubreuil et Vuillaume, 1975 pour le calcul de la lame d'eau écoulée moyenne annuelle $\mathrm{E}_{\mathrm{c}}$ (exprimée en $\mathrm{mm}$ ) est la suivante :

$E_{c}=0,47 \times P_{r}-33 \times \log (S)+0,54 \times D_{s}+A$

Avec: Ec: Apport annuel d'eau où écoulement annuel d'eau (mm); S: la superficie du bassin en $\mathrm{km}^{2}$;

$D s:$ la dénivelée spécifique $(\mathrm{m})$;

$A$ : le terme d'aptitude à l'écoulement, il est de $-175 \mathrm{~mm}$ pour des bassins avec des dépressions en guise de thalwegs. Cette méthode est limitée aux calculs des apports en année moyenne interannuelle.

\section{Résultats}

3.1. Des dynamiques d'occupation du sol dictées par des besoins alimentaires et des besoins en logements de la mégapole abidjanaise 3.1.1. Le sous bassin versant de la Djibi: un bassin fortement urbanisé

Le bassin versant de la Djibi était dominé en 1987 par les cultures vivrières et les jachères (figure 2) avec une superficie de $33 \mathrm{~km}^{2}(42 \%)$ de la superficie totale de ce bassin. En 2000, la superficie de cette classe passe à 26 $\mathrm{km}^{2}(34 \%)$, soit une réduction de $7 \mathrm{~km}^{2}$. Cette régression s'est faite au profit de l'habitat dont la superficie est passée de $24 \mathrm{~km}^{2}$ en 1987 à $31 \mathrm{~km}^{2}$ en 2000 par rapport à l'ensemble du bassin (tableau 3). L'espace habité (partie Nord des communes d'Abobo, Cocody et Bingerville) du bassin va continuer de s'étendre pour atteindre une superficie de $42 \mathrm{~km}^{2}$ en 2015 , soit $53 \%$ de la superficie totale du bassin. Quant aux cultures pérennes à dominance palmier à huile et hévéa associées à une forêt dégradée, elles ont connu une réduction de leur superficie de 1987 à 2015 , passant de $4 \mathrm{~km}^{2}$ à $0,60 \mathrm{~km}^{2}$ et de $18 \mathrm{~km}^{2}$ à $10 \mathrm{~km}^{2}$ respectivement pour le palmier à huile et l'hévéa, soit des régressions de $4 \mathrm{~km}^{2}$ et $7 \mathrm{~km}^{2}$, respectivement. 

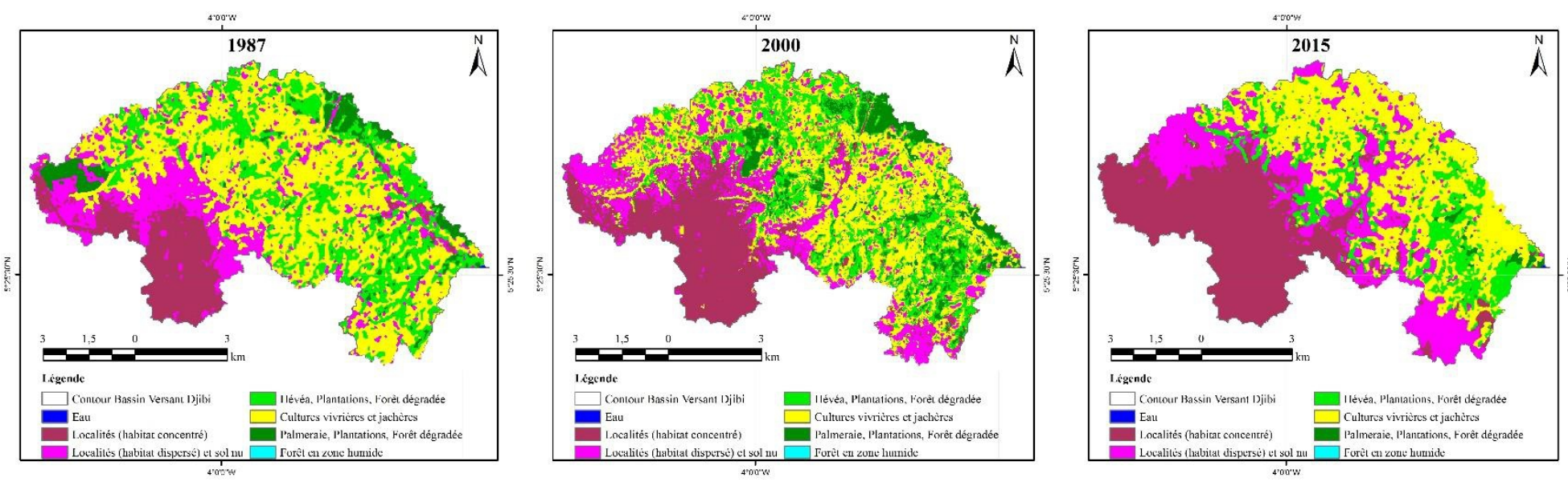

Figure 2 : Carte d'occupation du sol du sous bassin Djibi en 1987, 2000 et 2015

Tableau 3 : Pourcentage des classes d'occupation du sol du sous bassin Djibi en 1987, 2000 et 2015

\begin{tabular}{|c|c|c|c|c|c|c|c|c|c|}
\hline BV DJIBI & 1987 & 2000 & 2015 & $1987-2000$ & $2000-2015$ & $1987-2015$ & $1987-2000$ & $2000-2015$ & $1987-2015$ \\
\hline Classes OCS & \multicolumn{3}{|c|}{ Superficie $\left(\mathrm{km}^{2}\right)$ et Proportion $(\%)$} & \multicolumn{3}{|c|}{ Progression/Régression $\left(\mathrm{km}^{2}\right)$} & \multicolumn{3}{|c|}{ Taux d'évolution (\%) } \\
\hline EA & $0,02 \quad(0,03 \%)$ & $0,01 \quad(0,01 \%)$ & $0,02 \quad(0,01 \%)$ & $-0,01$ & 0,01 & 0 & -50 & 100 & 0 \\
\hline $\mathrm{HC}$ & $10,11(12,96 \%)$ & $17,65(22,63 \%)$ & $24,67 \quad(31,63 \%)$ & 7,54 & 7,02 & 14,56 & 74,58 & 39,77 & 144,01 \\
\hline $\mathrm{CVJ}$ & $32,63(41,83 \%)$ & $26,04(33,39 \%)$ & $25,45 \quad(32,63 \%)$ & $-6,59$ & $-0,59$ & $-7,18$ & $-20,2$ & $-2,27$ & -22 \\
\hline HD & $13,86(17,77 \%)$ & $13,13(16,83 \%)$ & $16,88 \quad(21,64 \%)$ & $-0,73$ & 3,75 & 3,02 & $-5,27$ & 28,56 & 21,79 \\
\hline FZH & $0,00 \quad(0,00 \%)$ & $0,00 \quad(0,00 \%)$ & $0,00 \quad(0,00 \%)$ & 0 & 0 & 0 & 0 & 0 & 0 \\
\hline PPFD & $4,16 \quad(5,33 \%)$ & $6,46 \quad(8,28 \%)$ & $0,60 \quad(0,77 \%)$ & 2,3 & $-5,86$ & $-3,56$ & 55,29 & $-90,71$ & $-85,58$ \\
\hline HPFD & $17,22(22,08 \%)$ & $14,71(18,86 \%)$ & $10,38 \quad(13,30 \%)$ & $-2,51$ & $-4,33$ & $-6,84$ & $-14,58$ & $-29,43$ & $-39,72$ \\
\hline TOTAL & $(100 \%)$ & $(100 \%)$ & $(100 \%)$ & - & - & - & - & - & - \\
\hline
\end{tabular}

$\mathrm{EA}=\mathrm{Eau} ; \mathrm{CVJ}=$ Cultures vivrières et jachères $; \mathrm{HC}=$ Habitats concentrés; $\mathrm{HD}=$ Habitats dispersés; HPFD= Hévéa + Plantations + Forêt dégradée $; \mathrm{PPFD}=$ Palmeraie + Plantations + Forêt dégradée $; \mathrm{FZH}=$ Forêt zone humide ; 


\subsubsection{Le bassin versant Bété: vers une colonisation de l'espace par le vivrier}

Le bassin versant de la Bété qui fait plus du double de celui de la Djibi en terme de superficie, est moins humanisé que son voisin (figure 3). La surface habitée

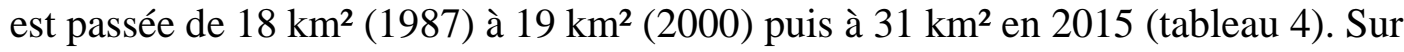
ce bassin, l'habitat s'est accru très rapidement de 1987 à 2015, les proportions passant de $9 \%$ à $15 \%$, soit un taux d'évolution de $67 \%$. Cette progression a connu un ralentissement en 2000 avec l'augmentation de la superficie de seulement de 1 $\mathrm{km}^{2}$ soit $19 \mathrm{~km}^{2}$ à cette date. La relique forestière $\left(0,04 \mathrm{~km}^{2}\right)$ qui existait a totalement disparu depuis 2000. Quant aux cultures pérennes palmier à huile et hévéa associés à la forêt dégradée, elles ont connu un dynamisme semblable à celui du bassin versant de la Djibi. Les superficies ont régressé de 1987 à 2015. Les surfaces couvertes par le palmier à huile et l'hévéa sont passées respectivement de $21 \mathrm{~km}^{2}$ en 1987 à $19 \mathrm{~km}^{2}$ en 2015 et de $98 \mathrm{~km}^{2}$ en 1987 à $29 \mathrm{~km}^{2}$, soient des baisses respectives de $2 \mathrm{~km}^{2}$ et $69 \mathrm{~km}^{2}$. La progression de la classe palmier à huile et forêt dégradée est remarquable sur les deux bassins versants. Mais, elle se fait sur des périodes différentes. Ce phénomène a été constaté de 2000 à 2015 sur le bassin versant de la Bété et de 1987 à 2000 sur celui de la Djibi. Cette augmentation était plus importante sur le bassin de la Bété soit une hausse de $7 \mathrm{~km}^{2}$. La superficie est passée de $12 \mathrm{~km}^{2}$

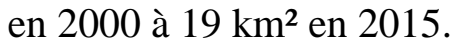



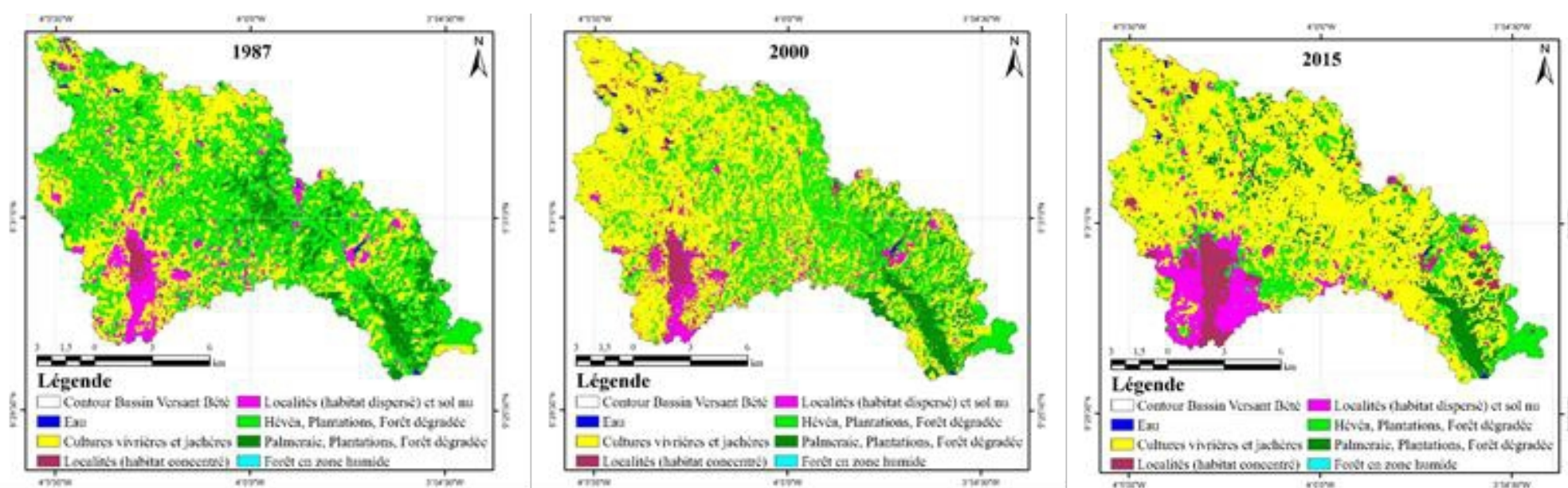

Figure 3 : Carte d'occupation du sol du sous bassin Bété en 1987, 2000 et 2015

Tableau 4 : Pourcentage des classes d'occupation du sol du sous bassin Bété en 1987, 2000 et 2015

\begin{tabular}{|c|c|c|c|c|c|c|c|c|c|}
\hline BV BÉTÉ & 1987 & 2000 & 2015 & $1987-2000$ & $2000-2015$ & $1987-2015$ & $1987-2000$ & $2000-2015$ & $1987-2015$ \\
\hline Classes OCS & \multicolumn{3}{|c|}{ Superficie $\left(\mathrm{km}^{2}\right)$ et Proportion $(\%)$} & \multicolumn{3}{|c|}{ Progression/ Régression $\left(\mathrm{km}^{2}\right)$} & \multicolumn{3}{|c|}{ Taux d'évolution (\%) } \\
\hline $\mathrm{HC}$ & $2,06 \quad(1 \%)$ & $10,04 \quad(4,87 \%)$ & $\begin{array}{ll}13,39 & (6,50 \%)\end{array}$ & 7,98 & 3,35 & 11,33 & 387,38 & 33,37 & 550 \\
\hline $\mathrm{HD}$ & $15,77(7,66 \%)$ & $8,54 \quad(4,15 \%)$ & $18,00 \quad(8,73 \%)$ & $-7,23$ & 9,46 & 2,23 & $-45,85$ & 110,77 & 14,14 \\
\hline FZH & $0,04 \quad(0,02 \%)$ & $(0,00 \%)$ & $(0,00 \%)$ & $-0,04$ & 0 & $-0,04$ & -100 & 0 & -100 \\
\hline PPFD & $20,73(10,06 \%)$ & $12,05 \quad(5,84 \%)$ & $18,67 \quad(9,06 \%)$ & $-8,68$ & 6,62 & $-2,06$ & $-41,87$ & 54,94 & $-9,94$ \\
\hline
\end{tabular}




\subsubsection{Evaluation des coefficients d'écoulement moyens annuels}

Les figures 4 et 5 montrent l'évolution des coefficients d'écoulement et de la pluviométrie moyenne annuelle des bassins de la Djibi et de la Bété sur la période 1960-2017. Les valeurs annuelles des coefficients d'écoulement varient de $7,77 \%$ à $31,33 \%$ avec un écart-type de 5,5\% et une moyenne égale à $19 \%$ sur le bassin versant de la Djibi. Par contre, sur le bassin versant de la Bété, ces coefficients d'écoulement calculés fluctuent entre 2,70\% et 29,78\% avec une moyenne de $14,59 \%$ et un écart-type de 5,8 \%. Pour chacun des bassins versants, les coefficients d'écoulement sont très peu contrastés. Les valeurs de pluviométrie moyenne annuelle sont corrélées aux coefficients d'écoulement. En 1987, pour une pluviométrie moyenne annuelle de 1792,6 mm, le coefficient d'écoulement était de 20,09\%. En 2000, pour une pluie de $1823 \mathrm{~mm}$, le coefficient d'écoulement était de 20,86\%. Et en 2015, le coefficient d'écoulement était de $21,13 \%$ pour une pluviométrie de 1849,3 $\mathrm{mm}$ à la Djibi. Sur la Bété (plus grand), le coefficient d'écoulement était de $13,19 \%$ pour une pluie moyenne annuelle de $1495 \mathrm{~mm}$ en 1987. Ce coefficient est passé à 15,68\% en 2000 pour une pluviométrie annuelle de $1623 \mathrm{~mm}$. Enfin en 2015, ce coefficient va augmenter et atteindre 17,07\% pour une pluviométrie de $1689,4 \mathrm{~mm}$.

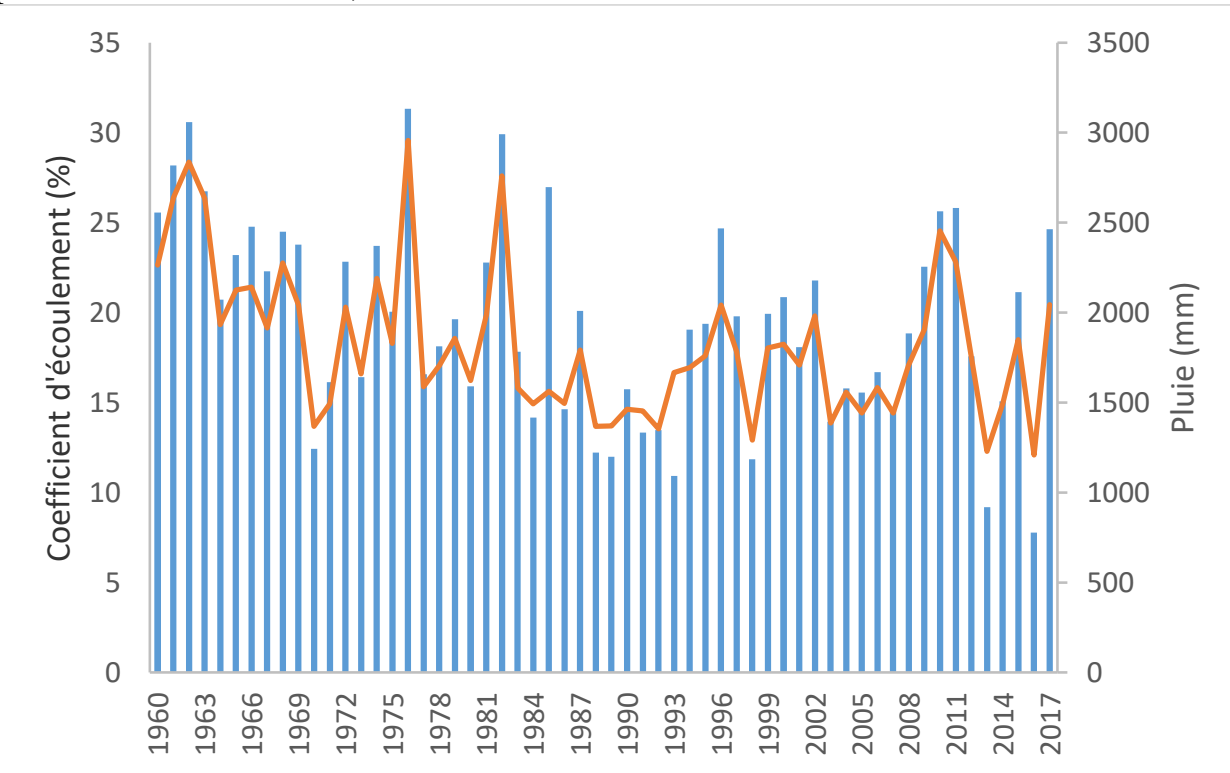

Figure 4: Evolution du coefficient d'écoulement annuel et de la pluviométrie moyenne annuelle du sous bassin versant de la Djibi 


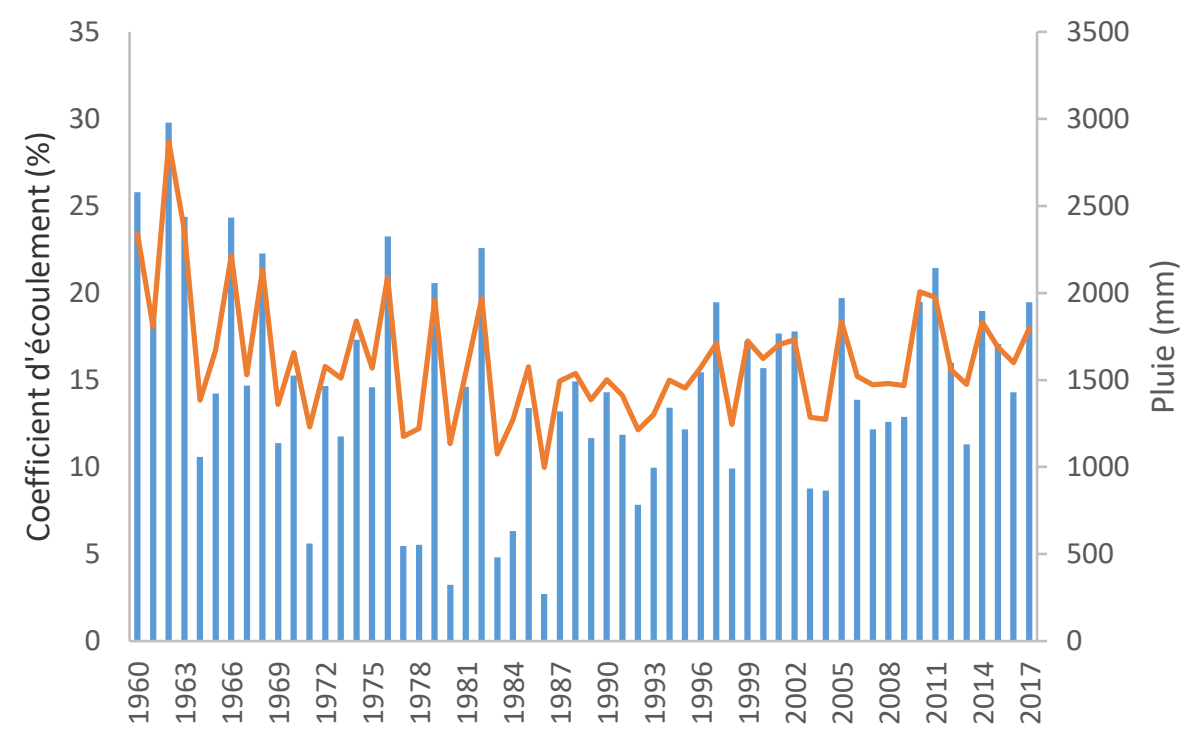

Figure 5: Evolution du coefficient d'écoulement annuel et de la pluviométrie moyenne annuelle du sous bassin versant de la Bété

\subsubsection{L'impact de l'anthropisation sur les écoulements}

L'une des conséquences directes de l'occupation du sol sur les écoulements est la variation des coefficients de rétention en eau en fonction du type d'occupation du sol.

L'évolution des coefficients de rétention et des coefficients d'écoulement des bassins versants de la Djibi et de la Bété en 1987, 2000 et 2015 est enregistrée sur les figures 6 et 7 puis dans le tableau 5. Les résultats s'apprécient différemment sur les deux bassins :

Pour le bassin versant de la Djibi (figure 6), la classe la plus dominante en termes de capacité de rétention d'une année à une autre est celle des cultures vivrières et des jachères. Le coefficient de rétention de cette classe diminue de $1987(41,83)$ à $2000(33,39)$ et $2015(32,63)$. Ensuite, vient la capacité de rétention de la classe hévéa sans toutefois modifier l'ordre de prédominance. Ainsi, on a: $(22,08)$ en 1987, $(18,86)$ en 2000 et $(13,30)$ en 2015. Contrairement au bassin de la Djibi (figure 7), la capacité de rétention des cultures vivrières et des jachères du bassin de la Bété est en nette progression. Elle est passée de 33,69 en 1987 à 55,87 en 2000, puis à 61,61 en 2015. Pourtant, la capacité de rétention de la classe hévéa a régressé de façon notable. Elle est passée de 47,35 en 1987 à 29,04 en 2000, puis à 13,86 en 2015. Seules, les capacités de rétention des classes habitat dispersé et palmier à huile de la zone d'étude ont subi des variations plus ou moins importantes. La classe habitat concentré indique que l'eau précipitée s'écoule en majorité sur les deux bassins versants étudiés du fait de leur coefficient nul. 
Le tableau 5 indique que tous les coefficients de rétention en eau sont inférieurs à 100 pour toutes les classes retenues. En 1987, le coefficient de rétention moyen du bassin versant de la Djibi est de 87,01 pour un coefficient d'écoulement de 20,09\%. En 2000, l'indice de capacité de rétention est en moyenne de 77,36 pour un coefficient d'écoulement de 20,86\%. En 2015, l'indice de capacité de rétention va diminuer pour atteindre 68,34 avec un coefficient moyen de $21,13 \%$. On constate donc une nette diminution des indices de capacité de rétention moyenne sur le bassin versant de la Djibi pendant que le coefficient d'écoulement augmente dans le temps. Sur le bassin versant de la Bété, on observe une légère augmentation des indices de capacité de rétention en eau. Ces indices de rétention moyenne sont passés de 98,8 en 1987 à 94,9 en 2000 pour en arriver à 93,26 en 2015. Les indices de rétention sont corrélés respectivement à des coefficients d'écoulement de 13,19\% en $1987,15,68 \%$ en 2000 et $17,07 \%$ en 2015 . Ainsi, ces résultats montrent que le bassin versant de la Djibi parait plus anthropisé, elle représente donc une zone propice aux écoulements. A l'inverse, le bassin versant de la Bété est à la limite de l'anthropisation. 

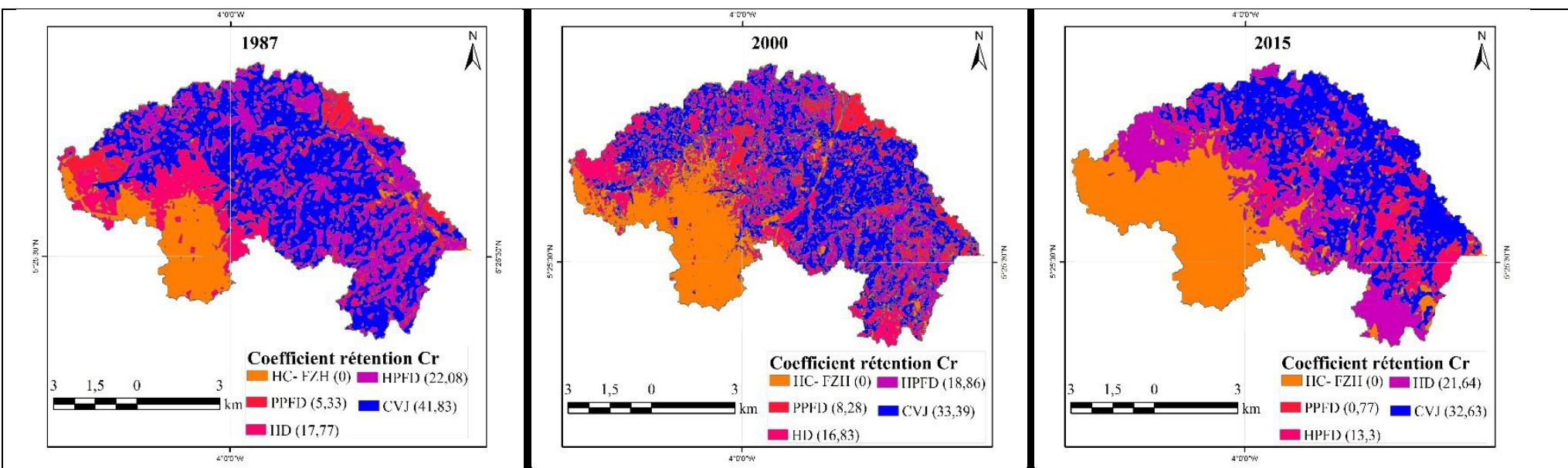

Figure 6 : Carte de répartition de l'indice de rétention en eau dans le bassin versant de la Djibi en 1987, 2000 et 2015
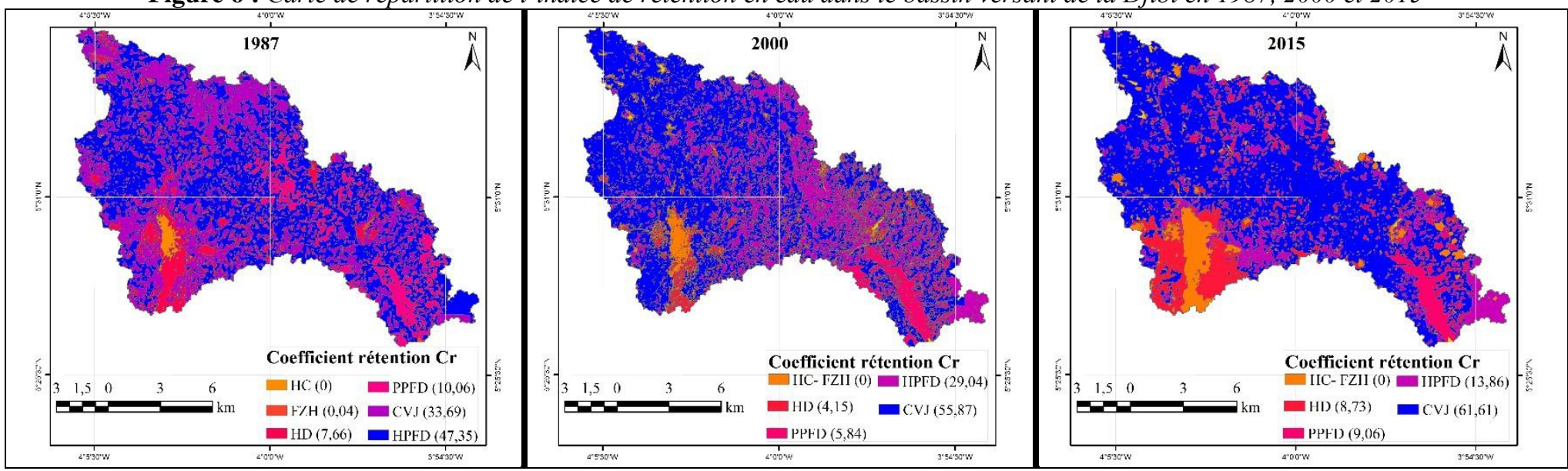

Figure 7 : Carte de répartition de l'indice de rétention en eau dans le bassin versant de la Bété en 1987, 2000 et 2015 
Tableau 5 : Evolution des coefficients de rétention $\mathrm{Cr}$ et d'écoulement $\mathrm{CE}$ dans la zone en 1987, 2000 et 2015

\begin{tabular}{|l|l|l|l|l|l|l|}
\hline \multirow{2}{*}{ Bassins } & \multicolumn{3}{|l|}{ Capacité de rétention en eau eau } & \multicolumn{3}{l|}{ Coefficient d'écoulement moyen annuel (\%) } \\
\cline { 2 - 7 } & 1987 & 2000 & 2015 & 1987 & 2000 & 2015 \\
\hline Djibi & 87,01 & 77,36 & 68,34 & 20,09 & 20,86 & 21,13 \\
\hline Bété & 98,8 & 94,9 & 93,26 & 13,19 & 15,68 & 17,07 \\
\hline
\end{tabular}

\section{Discussion}

L'analyse de la dynamique d'occupation du sol en 1987, 2000 et 2015 des sous bassins versants de la Djibi et de la Bété du système lagunaire Aghien montre une disparition quasi-totale de la forêt. La conversion rapide de la forêt en zone de culture, en jachère et en habitat est faite concomitamment avec une forte poussée démographique. En effet, l'évolution de la densité de la population va entraîner des besoins de logements. Ces besoins en logement se sont traduits depuis les années 1990 par la conquête des espaces péri-urbains par les particuliers et les opérateurs immobiliers privés. Dans cette même période, une baisse des superficies des cultures pérennes d'hévéa et de palmier à huile de $6 \%$ et $5 \%$ respectivement a été observée; cette dynamique régressive est due à l'extension spatiale du district d'Abidjan. Une étude menée par Sako (2013) sur l'impact de l'urbanisation sur la conservation du parc national du Banco à Abidjan a montré une occupation sensible de l'espace de cette forêt protégée de 7\% en 1998. Cependant, l'étalement urbain dans les bassins versants de la Djibi et de la Bété de 33\% et $14 \%$ entre 1987 et 2015, accentue l'artificialisation de ces milieux. De même, l'évaluation des écoulements moyens annuels a révélé une corrélation avec les séries pluviométriques. Les variations des coefficients d'écoulement sur la période d'observation semblent être liées aux fluctuations des averses et à l'anthropisation des bassins. La différence des coefficients d'écoulements surtout les faibles lames écoulées du bassin de la Bété par rapport à celui de la Djibi sont dues à la présence de l'arboriculture au stade adulte.

Ce résultat contraste avec ceux obtenus par Mahé et al., 2005, travaillant sur le comportement hydrologique des bassins soudano-sahéliens. Ces auteurs stipulent que plusieurs travaux ont enregistré simultanément une augmentation des coefficients de ruissellement dans les séries pluviométriques se caractérisant par une nette diminution des apports. Contrairement à cette assertion, les valeurs élevées du coefficient d'écoulement sur les bassins versants Djibi et Bété résultent de l'effet conjugué d'une augmentation des quantités pluviométriques annuelles et de l'artificialisation du milieu.

L'anthropisation modérée du bassin de la Bété est liée aux plantations agro-industrielles et villageoises qui ont atteint un stade adulte. L'augmentation des écoulements dans le bassin versant de la Djibi peut s'expliquer par la transformation des zones perméables agricoles (plantations d'hévéa et de palmier à huile) en zones imperméables urbaines (habitats et sols 
nus) d'une part, et par le développement des cultures à cycle court (cultures vivrières : maïs, manioc, maraichers, etc) d'autre part. Les cultures vivrières sont plus abondantes dans le bassin versant de la Djibi à cause de son contact direct avec les espaces urbanisés de la ville d'Abidjan où la demande du marché en vivriers est très forte. D’ailleurs la majorité des plantations de palmier à huile ont été remplacées par les cultures de manioc et de maïs. Ces cultures de subsistance n'ont pas une biomasse importante pour favoriser une importante infiltration des précipitations dans le sol. Selon Aké (2010) et Akognongbé et $a l$., 2014, l'accroissement des espaces de culture et des agglomérations engendrent une diminution progressive de l'étendue des surfaces boisées et la déstabilisation de la structure des sols. Cette dégradation du milieu accélère le ruissellement. Ainsi, l'augmentation de l'habitat, des champs et friches limite l'infiltration au profit de l'écoulement. Ces résultats s'accordent avec ceux obtenus par Yao et al., (2015) qui indiquent que l'urbanisation galopante contribue à réduire les zones d'infiltration des eaux. Les indices de rétention en eau calculés pour les bassins de la Djibi et de la Bété sont en dessous de 100 sur toute la chronique,témoignant ainsi du degré d'anthropisation de ces deux sous bassins. Dans ce cas, la capacité de rétention est faible entraînant un écoulement de surface important dont la principale conséquence est l'érosion. Ce résultat est en conformité avec ceux obtenus par Soro et al., (2014 ) dans une étude portant sur la dynamique d'occupation du sol dans la région des lacs qui ont montré que plus les sites sont anthropisés ou dégradés, plus l'écoulement de surface est important et les phénomènes d'érosion ont tendance à se mettre en place. Dans divers bassins de Côte d'Ivoire, plusieurs travaux (Noufé, 2011 ; Traoré et al., 2012, Koffi et al., 2014 ; et N'Go, 2015) ont quant à eux souligné que la dégradation du couvert végétal dans une région est le résultat d'un long processus de sélection naturelle sous l'action du climat, des conditions agroclimatiques, édaphiques et topographiques.

En somme, l'artificialisation du domaine d'étude en relation avec la dégradation du couvert végétal a affecté l'écoulement de surface

\section{Conclusion}

L'impact de la dynamique de l'occupation du sol sur les écoulements des rivières Djibi et Bété du littoral Ivoirien, a donc été évalué à partir du couplage par télédétection et SIG des images satellitaires d'une part, et par le calcul des écoulements moyens annuels à partir de la méthode de VuillaumeDubreuil d'autre part. Il ressort de l'analyse diachronique des données satellitaires Landsat Thematic Mapper (1987), Enhance Thematic Mapper Plus (2000) et Operational Land Imager (2015) que le bassin versant de la Djibi est fortement anthropisé. L'augmentation notable des cultures vivrières et des jachères dans la zone se ressent par la présence d'habitats et de 
populations. Avons nous constaté aussi dans les bassins versants Djibi et Bété, une conversion de la forêt à plus de $99 \%$ au profit des surfaces habitées et des cultures. Fort de ce constat, le changement opéré a un fort impact sur les coefficients d'écoulement et les capacités de rétention en eau. L'extension de l'habitat et des cultures s'est traduite par une nette augmentation des coefficients d'écoulement. L'artificialisation des bassins versants de la Djibi et de la Bété est marquée par de faibles valeurs de coefficient de rétention en dessous de 100. Dans l'ensemble, le bassin versant de la Djibi apparaît plus dégradé et plus anthropisé, avec des coefficients de rétention moins élevés que la Bété.

\section{Reconnaissances}

Au terme de cette étude, nous tenons à remercier les animateurs du programme PRESED (Partenariat rénové pour la Recherche au Service du Développement de la Côte d'Ivoire) et de l'IRD (Institut de Recherche pour le Développement) dans l'espace AMRUGE-C2D 2015-2017 pour avoir accepté de financer le projet AGHIEN auquel nous avons bénéficié.

\section{References:}

1. Aké E. G., (2010). Impacts de la variabilité climatique et des pressions anthropiques sur les ressources hydriques de la région de Bonoua. Thèse de doctorat de l'Université de Cocody, Côte d'Ivoire, 204 p.

2. Akognongbe A., D. Abdoulaye, E. W. Vissin et M. Bako, (2014). Dynamique de l'occupation du sol dans le bassin versant de l'Oueme à l'exutoire de Bétérou (Bénin). Afrique Science, vol. 10, no. 2, pp. 228-242.

3. Barima Y. S., N. Barbier, I. Bamba, D. Traoré, J. Lejoly et J. Bogaert, (2009). Dynamique paysagère en milieu de transition forêt-savane ivoirienne. Bois et Forêts des Tropiques, no 299 (1), pp. 15-25.

4. Bondy, J., (2015). Fonctionnement hydrologique et pollution en zone tropicale humide dans le bassin versant péri-urbain de la lagune Aghien. Rapport de stage de Master Eau, Université de Montpellier, France, $66 \mathrm{p}$.

5. Cappelaere B., L. Descroix and T. Lebel, (2009). The AMMA catch observing system in the cultivated Sahelian of South West Niger strategy, implementation and site conditions. Journal of Hydrology, 375 (1-2), pp. 34-51.

6. Cecchi J.-P., F. Gourdin, S. Koné, D. Corbin, A. Etienne, et Casenave, (2009). Les petits barrages du nord de la Côte d'Ivoire : inventaire et potentialités hydrologiques. Sécheresse, vol. 20, No. 1, pp. 112-122.

7. Chatelain C., (1996). Possibilités d'application de l'imagerie satellitaire à haute résolution pour l'étude des transformations de la 
végétation en Côte d'Ivoire forestière. Thèse de doctorat de l'Université de Genève, Suisse, $158 \mathrm{p}$.

8. Coulibaly L., K. H. Kouassi, G. E. Soro, et I. Savane, (2016). Analyse du processus de savanisation du nord de la Côte d'Ivoire par télédetection : cas du département de Ferkessédougou. International Journal Innovation and Applied Studies, vol. 17, no.1, pp. 136-143.

9. Dao A., (2013). Caractérisation des composantes du cycle de l'eau et processus de production de l'écoulement: cas du bassin versant transfrontalier du Kolondièba au Sud du Mali en milieu tropical de socle. Thèse de doctorat de l'Université Nangui Abrogoua, Côte d'Ivoire, $184 \mathrm{p}$.

10. Dubreuil P., G. Villaume, (1975). Influence du milieu physicoclimatique sur l'écoulement de petits bassins intertropicaux. Int. Assoc. Sci. Hydrol., no. 177, pp. 205-215.

11. Jourda J.-P., (1987). Contribution à l'étude géologique et hydrogéologique de la région du grand Abidjan (Côte d'Ivoire). Géologie Appliquée, Université Scientifique et Médicale de Grenoble, Suisse, $299 \mathrm{p}$.

12. Koffi K. J.-P., A. Y. N'Go, M. K. Yéo, D. Koné et I. Savané, (2014). Détermination des périmètres de protection de la Lagune Aghien par le calcul du temps de transfert de l'eau jusqu'à la lagune. Larhyss Journal, no. 19, pp. 19-35.

13. Kouassi K. J.-L., (2014). Suivi de la dynamique d'occupation du sol à l'aide de l'imagerie satellitaire et des sysrèmes d'informations géographiques : cas de la direction régionale des eaux et forêts de Yamoussoukro (Côte d'Ivoire). Mémoire du Diplôme d'Agronomie Approfondie (DAA) à l'Insitut National Polytechnique de Yamoussokro, $52 \mathrm{p}$.

14. Mahe G., J.-E. Paturel, E.B. Servat, D. C. Conway and A. B. Dezetterb, (2005). The impact of land use change on soil water holding capacity and river flow modelling in the Nakambe River, Burkina-Faso. Journal of hydrology, vol 300, pp 33-43.

15. N'go Y. A., (2015). Hydrologie et dynamique de l'état de surface des terres dans le Sud-Ouest de la Côte d'Ivoire: impacts et moteurs de dégradation. Doctorat d'état ès Sciences Naturelles de l'Université Nangui Abrogoua en Sciences et Gestion de l'Environnemen, Côte d'Ivoire $198 \mathrm{p}$.

16. Noufé D., (2011). Changements hydroclimatiques et transformations de l'agriculture: l'exemple des paysanneries de l'Est de la Côte d'Ivoire. Thèse de Doctorat de l'Université Paris 1 PanthéonSorbonne, France, 375 p.

17. Sako N., G. Beltrando, G. L. Atta, H. D. N'Da et T. Brou, (2013). 
Dynamique forestière et pression urbaine dans le Parc national du Banco (Abidjan, Côte d'Ivoire). Controverses environnementales, vol. 13, no. 2, pp. 1-23.

18. Soro, N., 2004. Variabilité du régime pluviométrique du Sud de la Côte d'Ivoire et son impact sur l'alimentation de la nappe d'Abidjan. Sud Sciences \& Technologies.

19. Soro G., (2010). Evaluation quantitative et qualitative des ressources en eaux souterraines dans la région des lacs (Centre de la Côte d'Ivoire) : hydrogéologie et hydrochimie des aquifères discontinus du district de yamoussoukro et du département de Tiébissou. Thèse de doctorat de l'Université de Cocody-Abidjan, Côte d'Ivoire, 254 p.

20. Soro G., E. K. Ahoussi, E. K. Kouadio, T. D. Soro, S. Oulare, M. B. Saley et N. Soro, (2014). La dynamique de l'occupation du sol dans la région des Lacs (Centre de la Côte d'Ivoire). Afrique science, vol. 10, no. 3, pp. 146-160.

21. Soro N., T. Lasm, B. H. Kouadio, G. Soro et K. E. Ahoussi, (2006). Variabilité du régime pluviométrique du Sud de la Côte d'Ivoire et son impact sur l'alimentation de la nappe d'Abidjan. Sud sciences et technologies, no. 14, pp. 30-40.

22. Soro T. D., B. D. Kouakou, E. A. Kouassi, G. Soro, A. M. Kouassi, K. E. Kouadio, M.-S. O.Yéi et N. Soro, (2013). Hydroclimatologie et dynamique de l'occupation du sol du bassin versant du haut Bandama à Tortya (Nord de la Côte d'Ivoire). VertigO, vol. 13, no. 3, pp. 1-22.

23. Tra Bi Z. A., (2013). Etude de l'impact des activités anthropiques et de la variabilité climatique sur la végétation et les usages des sols, par utilisation de la télédétection et des statistiques agricoles, sur le bassin versant du Bouregreg (Maroc), Thèse de doctorat Université Hydrosciences Montpellier, France, 190 p.

24. Traoré A., G. Soro, E. K. Kouadio, B. S. Bamba, M. S. Oga, et N. Soro, (2012). Evaluation des paramètres physiques, chimiques et bactériologiques des eaux d'une lagune tropicale en période d'étiage: la lagune Aghien (Côte d'Ivoire). Int. J. Biol. Chem. Sci., vol. 6, no. 6, pp. 7048-7058.

25. Vissin W., 2007. Impacts de la variabilité climatique et de la dynamique des états de surface sur les écoulements du bassin versant béninois du fleuve Niger, Thèse de Doctorat de l'Université de Bourgogne, France, $286 \mathrm{p}$.

26. Yao B. A., K. I. Kouamé, K. A. Kouassi, K. Kouadio, B. T. A. Goula et I. Savané, (2015). Estimation de la recharge d'une nappe côtière en zone tropicale humide: cas de la nappe du continental Terminal d'Abidjan (Côte d'Ivoire). International Journal Innovation and Applied Studies, vol. 12, no. 4, pp. 888-898. 